\title{
Fundamental principles of an effective diabetic retinopathy screening program
}

\author{
Paolo Lanzetta ${ }^{1,2}$ (1) Valentina Sarao ${ }^{1,2} \cdot$ Peter H. Scanlon ${ }^{3} \cdot$ Jane Barratt $^{4} \cdot$ Massimo Porta $^{5} \cdot$ Francesco Bandello $^{6}$. \\ Anat Loewenstein ${ }^{7}$ on behalf of the Vision Academy
}

Received: 17 December 2019 / Accepted: 14 February 2020 / Published online: 28 March 2020

(c) The Author(s) 2020, corrected publication 2020

\begin{abstract}
Background Diabetic retinopathy (DR) is the leading cause of blindness among working-age adults worldwide. Early detection and treatment are necessary to forestall vision loss from DR.

Methods A working group of ophthalmic and diabetes experts was established to develop a consensus on the key principles of an effective DR screening program. Recommendations are based on analysis of a structured literature review.

Results The recommendations for implementing an effective DR screening program are: (1) Examination methods must be suitable for the screening region, and DR classification/grading systems must be systematic and uniformly applied. Twofield retinal imaging is sufficient for DR screening and is preferable to seven-field imaging, and referable DR should be well defined and reliably identifiable by qualified screening staff; (2) in many countries/regions, screening can and should take place outside the ophthalmology clinic; (3) screening staff should be accredited and show evidence of ongoing training; (4) screening programs should adhere to relevant national quality assurance standards; (5) studies that use uniform definitions of risk to determine optimum risk-based screening intervals are required; (6) technology infrastructure should be in place to ensure that high-quality images can be stored securely to protect patient information; (7) although screening for diabetic macular edema (DME) in conjunction with DR evaluations may have merit, there is currently insufficient evidence to support implementation of programs solely for DME screening.
\end{abstract}

Conclusion Use of these recommendations may yield more effective DR screening programs that reduce the risk of vision loss worldwide.

Keywords Diabetic retinopathy screening $\cdot$ Telemedicine $\cdot$ Evidence-based recommendations

This article belongs to the topical collection Eye Complications of Diabetes, managed by Giuseppe Querques.

The Vision Academy is a global group of ophthalmologists, convened by Bayer to provide guidance for best clinical practice through collective expertise in areas of controversy or with insufficient conclusive evidence. The Vision Academy Steering Committee advised on the initial publication concept, and this manuscript is based on their discussions around the subject area. Members and their affiliations are listed in Acknowledgements.

Paolo Lanzetta

paolo.lanzetta@uniud.it

1 Department of Medicine - Ophthalmology, University of Udine, Piazzale S. Maria della Misericordia, 33100 Udine, Italy

2 Istituto Europeo di Microchirurgia Oculare (IEMO), Udine, Italy

3 Gloucestershire Hospitals NHS Foundation Trust, Gloucester, UK

\section{Introduction}

Diabetic retinopathy (DR) is a well-known complication of diabetes and the main cause of blindness among workingage adults [1]. Timely detection and treatment of DR can prevent blindness, but many people with this condition are not diagnosed early enough to be treated effectively. People

4 International Federation on Ageing, Toronto, Canada

5 Department of Medical Sciences, University of Turin, Turin, Italy

6 San Raffaele Scientific Institute, Milan, Italy

7 Department of Ophthalmology Tel Aviv Medical Center, and Sackler Faculty of Medicine, Tel Aviv University, Tel Aviv, Israel 
with sight-threatening DR (STDR) are often asymptomatic during the period in which the condition is treatable by photocoagulation or intravitreal therapy [2]. Therefore, community-wide education and implementation of effective programs for DR screening are needed.

Numerous screening programs have been established throughout the world; these usually involve assessment and grading of the eyes of patients with diabetes, and referral of those with STDR to an ophthalmologist. The principles and considerations for screening programs, proposed by Wilson and Jungner on behalf of the World Health Organization in 1968 [3], have been adopted widely in practice, including in the English National Health Service (NHS) Diabetic Eye Screening Programme [4]. Since the implementation of this program in 2003, diabetic retinopathy/maculopathy is no longer the leading cause of blindness in the working population in England [4]. The International Council of Ophthalmology published the 2017 Guidelines for Diabetic Eye Care, which includes a guide to DR screening as well as follow-up and management based on different resource settings [5]. A successfully implemented screening program should benefit patients by increasing awareness of the importance of regular monitoring and providing a prompt referral to an ophthalmologist for screen-positive DR, to ultimately reduce the risk of sight loss and preserve patient function and quality of life [6-8].

Although established screening programs have reduced the risk of sight loss among people with diabetes worldwide, consensus among experts on effective screening in DR is lacking. Therefore, a working group of ophthalmic and diabetes experts was established and convened through the Vision Academy, a Bayer educational initiative, to propose evidence-based recommendations for screening for DR. This review will present these recommendations, assess unmet needs and identify areas for further investigation.

\section{Methods}

\section{Searches}

A structured literature search with predetermined search terms and inclusion/exclusion criteria was undertaken to identify studies worldwide that address the effectiveness of DR screening programs and screening tools. An initial search of MEDLINE/PubMed was performed on March 8, 2018, using the following search terms: "diabetic retinopathy" OR "diabetic macular edema" OR DME OR "diabetic macular oedema" OR DMO AND "screening program" OR screening OR "teleretinal screening" OR telemedicine OR teleophthalmology.
After screening study titles for relevance, abstracts were read, and studies that involved the following were included: (1) patients with diabetes, (2) a sample size of $>200$ patients and (3) quality or cost-effectiveness of DR screening programs or telemedicine systems. Only studies published in the preceding 10 years (to March 8,2018 ) were included, and those with the greatest scientific impact (average of $\geq 3$ citations per year) were prioritized. No study exclusions were made on the basis of gender, age, disease severity, presence of comorbidities, socioeconomic status or geographic region.

After screening titles and abstracts, the full text from 231 articles were examined in detail and 89 articles of interest were identified for the review.

\section{Results}

\section{Standard imaging techniques}

As the pathology of DR includes characteristic microvascular lesions detectable on fundus examination, obtaining ophthalmic images is the primary method of screening for DR. The most typical imaging method used is retinal fundus photography (FP), but dilated slit-lamp biomicroscopy may be another option. Results can be assessed at the point of care or in a telemedicine setting. Imaging is performed by trained personnel (e.g., technicians, nurses, family physicians, optometrists or endocrinologists), and results can be evaluated manually by certified graders/readers (e.g., ophthalmologists, retina specialists or trained technicians) or automatically by image analysis algorithms.

Seven-field stereoscopic FP was recognized as the reference standard for assessing effectiveness of DR screening programs in the Early Treatment Diabetic Retinopathy Study (ETDRS) [4]. However, in a review of 45 studies, researchers noted that the most commonly used imaging method in DR screening was two-field fundus imaging [9].

Both mydriatic and non-mydriatic approaches are common in DR screening programs. Induction of mydriasis may improve specificity of DR detection, but involves longer examination time and greater patient discomfort. In a study of community-based screening for STDR, Baeza et al. (2009) determined that imaging one non-stereoscopic field with a non-mydriatic camera (NMC) was comparable in sensitivity and specificity to seven-field standard stereoscopic imaging, as long as mydriasis was performed. If NMC screening without mydriasis was performed, the percentage of patients referred to ophthalmologists increased from 5 to $15 \%$ because of ungradable photographs [10]. In a metaanalysis including data published up to June 2009, variations in mydriatic status did not significantly affect sensitivity or 
specificity of DR detection [11]. However, subsequent metaanalyses do not support this. The authors in India concluded that non-mydriatic digital imaging had low sensitivity and resulted in a high rate of ungradable images, particularly in a dark iris population [12]. Similar results were obtained in a pilot telescreening study in Italy that involved 22,466 patients [13]. In a meta-analysis of digital imaging-based telemedicine for DR screening, the researchers found that diagnostic accuracy was higher when images were obtained when mydriasis was performed versus when it was not, especially when a wide angle was used [14].

\section{Alternative imaging techniques}

Non-mydriatic ultra-wide-field (UWF) imaging and optical coherence tomography (OCT) may be applicable in future DR screening efforts; work is ongoing to establish the validity and cost-effectiveness of these tools at the point of care and in a telemedicine setting [15-19].

UWF applies the principles of scanning laser ophthalmoscopy (SLO) with an ellipsoid mirror to capture a $200^{\circ}$ image versus the $45^{\circ}-50^{\circ}$ image obtained with standard FP. This permits imaging of the peripheral retina and observation of DR lesions that might otherwise be missed [16]. An example of an image obtained using UWF FP is shown in Fig. 1. A telemedicine program in which trained non-physicians performed UWF SLO imaging and immediate grading of minimal DR was found to have good sensitivity and specificity [18]. Compared with the reading center evaluation, real-time image evaluation had sensitivity of 0.95 (95\% confidence interval [CI] 0.94-0.97) and specificity of 0.84 (95\% CI 0.82-0.85) for minimal DR, and for referable DR

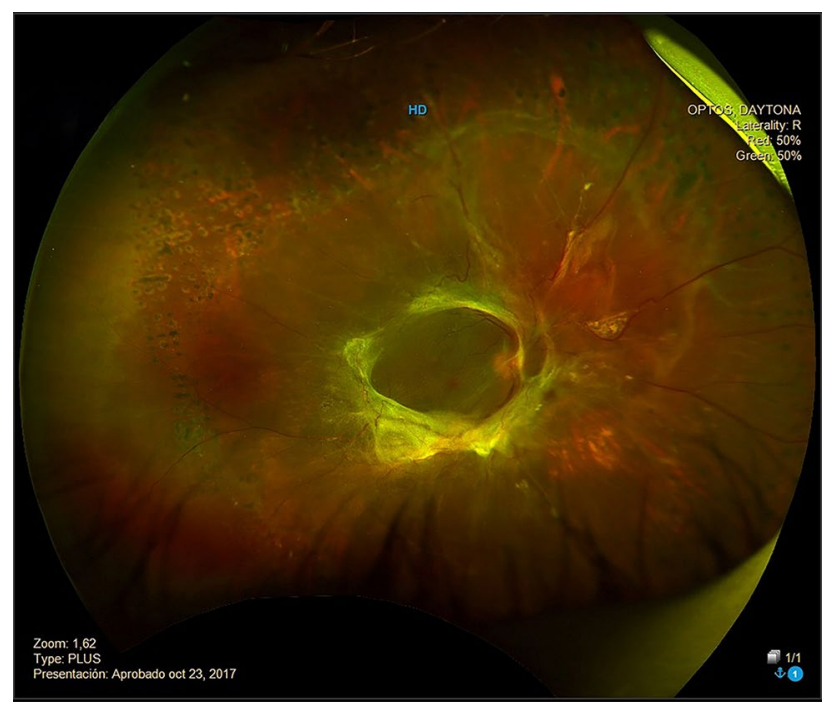

Fig. 1 Example of a UWF fundus image showing significant fibrosis due to proliferative diabetic retinopathy
(RDR) had sensitivity of 0.99 (95\% CI 0.97-1.00) and specificity of 0.76 (95\% CI $0.75-0.78)$ [18]. In addition, there were relatively few ungradable images (ungradable rate of $5.3 \%$ per eye) [18].

In large-scale DR teleophthalmology programs, results from non-mydriatic UWF SLO imaging were found to be superior to results from non-mydriatic multifield FP [17]. Specifically, UWF imaging reduced the number of ungradable eyes by $81 \%$, increased DR detection twofold and enabled detection of predominantly peripheral lesions (which are suggestive of severe DR) in almost $10 \%$ of patients [17]. In a study that compared UWF with clinical examination alone, UWF imaging allowed for earlier diagnosis of more patients with higher-grade DR [20].

In a private multispecialty hospital in the United Arab Emirates, imaging with a UWF fundus camera by nursing personnel in the endocrinology department was an effective form of telemedicine for DR screening [19]. This method ameliorated the burden of screening for DR in the retina clinic and improved the rate of early detection of DR [19]. Although an early UWF SLO study required more time for image analysis, yielded a higher rate of ungradable eyes and failed to detect some foveal microaneurysms (MA) compared to a digital camera [21], a more recent study demonstrated that the peripheral lesions detected through UWF imaging showed that the level of DR was more severe in $10 \%$ of patients than shown by non-mydriatic multifield fundus imaging [17].

The value of OCT in conjunction with FP for DR screening is currently unclear. Unlike two-dimensional FP, OCT provides volume and thickness data with three-dimensional visualization of pathological changes related to DME and can be used to confirm or rule out DME. In cases where DME is confirmed, OCT provides objective quantitative data to help guide treatment decisions and management of DME. Disadvantages of OCT include equipment costs and the need for a highly skilled operator to interpret images and determine imaging artifacts [15]. OCT is not advocated in primary screening because the price of the OCT equipment makes it highly unlikely that it will be cost-effective, given that in most screening studies at least $65 \%$ of the population with diabetes do not have DR. Advocates for the use of OCT currently recommend that it is only used to confirm DME when there is evidence of diabetic maculopathy from standard digital color photographs and two-dimensional markers. This approach has been shown to be cost-effective [22, 23].

Smartphone-based imaging tools may be useful for DR screening programs in which cost and availability of trained eye care personnel are barriers to implementation [24, 25]. However, no handheld device has been found to have comparable sensitivity and specificity to seven-field stereoscopic photography in detection of STDR. Rajalakshmi et al. [26] compared the "fundus on phone" (FOP) smartphone-based retinal camera with seven-field digital retinal photography 
and noted that the modalities yielded the same results for $92.7 \%$ of patients (kappa, 0.90). These authors suggested that the FOP camera was effective for screening and diagnosis of DR and STDR [26]. However, they noted that all patients underwent mydriasis in this study and that the image quality of the reference standard was superior to that of the FOP system. In this study, the smartphone was fixed and the patient's head was secured by using a slit-lamp chin rest. This simple variation may significantly improve results from the use of smartphone technology because it reduces any image blurring caused by movement of the operator and/ or the patient. More research is needed in terms of multisite trialing, measurement of the impact of smartphone-based DR assessment on clinical workflow and determination of the effects of this technology on health outcomes of screened patients [24].

\section{Functional assessments}

At present, visual acuity is the most widely used functional measure in routine DR screening. Loss of visual acuity may occur at different stages of DR, but visual acuity alone is not a reliable measure for predicting DR. For example, in a study of 1549 patients with diabetes, the sensitivity and specificity of using subnormal vision to screen for STDR were $33.4 \%$ and $85.9 \%$, respectively [27], which are well below recommended thresholds of $80 \%$ and $95 \%$, respectively [28].
Measuring visual acuity alone may therefore not be sensitive enough to detect early DR so should only be used in conjunction with imaging methods during screening. However, as stereoscopic imaging facilities may not be available in all centers, in some circumstances it could be necessary to rely only on visual acuity measures for detecting potential DR. Other measures (including contrast sensitivity, dark adaptation and electrophysiologic parameters) can be helpful endpoints in translational research [29]. However, more research is warranted to determine whether measures other than visual acuity have any utility in DR screening [29].

\section{Grading disease severity}

Different scales are used for grading the severity of DR. The ETDRS scale has 11 grades, from no disease to advanced proliferative DR (PDR) [30]. Although this scale is useful for research purposes, it is more complex than what is needed for DR screening programs, where the goal is to identify patients for referral and treatment. Accordingly, grading systems that have fewer grades and therefore simplify classification have been developed for DR screening. Typically, these scales acknowledge the four stages of DR: mild non-proliferative DR (NPDR), moderate NPDR, severe NPDR and PDR [31]. Examples and definitions of the different grades are shown in Table 1 [5, 32, 33].

In a meta-analysis, Bragge et al. (2011) noted inconsistencies among DR classification schemes used as a basis for

Table 1 Examples of grading scales for DR

\begin{tabular}{|c|c|c|c|}
\hline ETDRS grading (level) & ICO grading & AAO grading & RCOphth grading \\
\hline No disease (10) & No abnormalities & No abnormalities & No disease \\
\hline MA only (20) & $\begin{array}{l}\text { Mild NPDR } \\
\text { MA only }\end{array}$ & $\begin{array}{l}\text { Mild NPDR } \\
\text { MA only }\end{array}$ & Low risk \\
\hline Mild NPDR (35) & $\begin{array}{l}\text { Moderate NPDR } \\
\text { MA + other signs, excluding those indicating } \\
\text { severe NPDR }\end{array}$ & $\begin{array}{l}\text { Moderate NPDR } \\
\text { MA + other signs, but none defining severe } \\
\text { NPDR }\end{array}$ & - \\
\hline Moderate NPDR (43) & - & - & High risk \\
\hline Moderately severe NPDR (47) & - & - & - \\
\hline Severe NPDR (53A-D) & $\begin{array}{l}\text { Severe NPDR } \\
>20 \text { intraretinal hemorrhages in all quadrants } \\
\text { Venous beading in two or more quadrants } \\
\text { IRMA in one or more quadrant }\end{array}$ & $\begin{array}{l}\text { Severe NPDR } \\
\text { Intraretinal hemorrhages in all quadrants } \\
\text { Venous beading in two or more quadrants } \\
\text { IRMA in one or more quadrant }\end{array}$ & - \\
\hline Very severe NPDR (53E) & - & - & - \\
\hline Mild PDR (61) & $\begin{array}{l}\text { PDR } \\
\text { Neovascularization and/or vitreous/prereti- } \\
\text { nal hemorrhage }\end{array}$ & $\begin{array}{l}\text { PDR } \\
\text { Neovascularization and/or vitreous/prereti- } \\
\text { nal hemorrhage }\end{array}$ & PDR \\
\hline Moderate PDR (65) & - & - & - \\
\hline High-risk PDR $(71,75)$ & - & - & - \\
\hline Advanced PDR $(81,85)$ & - & - & - \\
\hline
\end{tabular}

$A A O$ American Academy of Ophthalmology, DR diabetic retinopathy, ETDRS Early Treatment Diabetic Retinopathy Study, ICO International Council of Ophthalmology, IRMA intraretinal microvascular abnormalities, MA microaneurysms, NPDR non-proliferative diabetic retinopathy, $P D R$ proliferative diabetic retinopathy, $R C O p h t h$ Royal College of Ophthalmologists 
referral [11]. According to the International Clinical DR and DME Disease Severity Scales, developed by the Global Diabetic Retinopathy Project Task Force on behalf of the American Academy of Ophthalmology (AAO) in 2002, any level of retinopathy more severe than mild retinopathy may warrant examination by an ophthalmologist [32]. This means that, according to the AAO definition of mild NPDR, a referral is needed for anything more than "microaneurysms only" (ETDRS level > 20) [32]. However, in the Royal College of Ophthalmologists (RCOphth) grading system, "low risk" equates to the AAO definition of mild NPDR, but the RCOphth referral for "high risk" is recommended at a more advanced stage (ETDRS level of 43) than the equivalent AAO referral (Table 1) [5, 32, 33]. These observations underscore that a universal feature-based classification/grading system for discerning DR is lacking.

\section{Telemedicine-based screening}

Telemedicine is essentially the ability to locally capture digital data (including images) and send the files to a centralized location for evaluation. Telemedicine systems based on digital fundus imaging offer a feasible and efficient means to screen patients who are not being reached by screening efforts in the specialist's office [2]. A review of the literature confirmed that telemedicine has the potential to modify patient behaviors, which would contribute to diabetes control and prevention [34]. Telemedicine screening may take place at a diabetes clinic; in the primary care offices of a physician, optometrist or pharmacist; at university hospitals; or in mobile units.

In a randomized controlled trial, Mansberger et al. [35] compared the effectiveness of DR telescreening with an NMC versus traditional surveillance by an eye care provider. The authors determined that patients in the telemedicine group were more likely to undergo DR screening in the first year of enrollment [35]. Two years into the study, telemedicine-based screening was offered to all patients [36]. The authors' 5-year findings indicate that telemedicine is an effective means of initial DR screening and monitoring for DR progression [36]. In a hypothetical cohort of unscreened patients with type 2 diabetes, a telemedicine-based DR screening model with trained technicians had lower costs than a physician-based model and produced similar qualityadjusted life years (QALYs) from a societal perspective [37].

DR screening with a mobile NMC can be beneficial for reaching patients who otherwise would not be included in a recommended screening program [38]. Cuadros and Bresnick [39] noted that patients tend not to seek annual DR screening and suggested incorporating telemedicine screening into primary care practices; these authors applied EyePACS, a Web-based means of retinopathy grading. Results from an Australian study showed that general practice-based DR screening is effective, enabling improved recording of screening outcomes and facilitating better follow-up of patients with RDR [40]. In a review, Das et al. [41] determined that DR is highly suitable for telemedicine because it saves time and minimizes lost income for the patient. DR telescreening can also provide cost savings for health systems depending on the population being screened (see the "Cost-effectiveness of screening" section); for example, a review highlighted that remote and underserved areas benefited most, with one rural teleophthalmology program resulting in savings of $\$ 150$ per patient over 7 years [42].

Based on a literature review, Surendran and Raman [43] concluded that telescreening for DR is cost-effective, accurate and reliable, and that digital imaging systems are safe and effective alternatives to dilated indirect ophthalmoscopy coupled with biomicroscopy or stereoscopic FP. Different countries have different health service systems in place, and financial support for screening programs can be difficult to secure. Charitable sources, such as the Queen Elizabeth Diamond Jubilee Trust, provide grants to fund DR screening programs in less developed nations [44].

Pareja-Ríos et al. [45] reviewed 8 years of experience in teleophthalmology screening (Retisalud program; Canary Islands) and found that the number of patients screened per year increased steadily; the waiting times for image assessment decreased, and the ability of family doctors to correctly interpret retinographies improved progressively. In addition, the rate of ungradable retinographies decreased-partly owing to incorporation of mydriasis - and the percentage of images graded as normal increased [45]. Moreover, cases of severe NPDR and PDR constituted 14\% initially and 3\% at the end of the study. These findings underscore the fact that the full benefits of a DR screening program can take several years to develop [45].

\section{Staff accreditation and training}

An effective DR screening program should include an accreditation system and require staff to demonstrate evidence of ongoing training. In the English NHS Diabetic Eye Screening Programme, all screeners and graders must show evidence of continuing professional development and take monthly external quality assurance tests. An international version of the accreditation and tests are also available for those working outside the UK [4].

Trained primary care physicians have been found to grade retinal photographs with acceptable accuracy when compared with ophthalmologists. In a study by Farley et al. (2008), the trained clinicians failed to refer $35(10.2 \%)$ of the 344 patients that the ophthalmologist believed needed referral, which was concluded to be reasonable [46]. Romero 
et al. (2010) found grading concordance in terms of DR, DME and macular lesions between family physicians and reference ophthalmologists; the authors concluded that involving family physicians in DR screening can be effective [47]. In a retrospective, cross-sectional study in Singapore, trained non-physician graders proved to be a satisfactory and cost-effective alternative to family physicians for detecting DR [48].

\section{IT infrastructure}

DR screening programs should be supported by a strong IT infrastructure that safeguards patient data and effectively balances image quality and file size. A typical DR screening program will require approximately 80,000 images to be stored per year. Although file size used to be a significant constraint [49], more recently file storage technology has improved and all files can be stored on a single server. The English NHS Diabetic Eye Screening Programme recommends that images captured are compressed to 1 to $2 \mathrm{MB}$ [4].

\section{Automated disease detection}

The diagnostic performance of artificial intelligence (AI)based software for DR detection has been evaluated in several studies (Table 2). Deep learning (DL) is an AI-based application whereby convolutional neural networks (CNNs) train themselves to interpret images by iterative analysis and comparison of the output with a reference standard (i.e., diagnosis by human graders). The process is repeated by the CNN until the diagnostic output agrees with the reference standard. DL algorithms have been developed for the automated detection of DR lesions from color fundus photographs - these systems are being implemented in screening programs and have the potential to reduce screening costs and increase service efficiency in healthcare economies in the developed world and to aid delivery of DR screening in developing and remote settings [16, 50-54]. Retinal fundus imaging with an NMC and automated grading take only 10 minutes, can be performed at the point of care and may obviate a separate visit to an ophthalmologist [55]. Automated analysis of OCT images through use of DL is being explored in a collaborative project between Moorfields Eye Hospital and Google DeepMind [56], and in other retinal centers of excellence [57].

Fleming et al. (2010) affirmed that including automated algorithms to identify RDR lesions in digital fundus images is likely to be a cost-effective adjunct to manual grading. By adding algorithms for detection of exudates and blot hemorrhages to an MA detection algorithm, a significant increase in sensitivity of RDR detection was achieved, without there being an increase in the manual grading workload [58]. The automated software detected $100 \%$ of patients with proliferative or referable/observable background retinopathy and would reduce workload by $36.3 \%$ compared with entirely manual grading [59].

\section{Quality assurance}

Service quality assurance reduces the probability of error and risk and helps professionals and organizations improve over time. Methods of quality assurance include post hoc evaluations of cost-effectiveness and accuracy of image grading [60]. In a South African study that included 261 ophthalmologists and optometrists, the Scottish Diabetic Retinopathy Grading Scheme was chosen because simplicity and clear cutoffs for referrals are vital for public sector eye clinics that have a high number of patients and limited resources [60]. The participants graded 90 retinal photographs as an external test of quality assurance, and the findings were: large disparities in grader performance; a general lack of specificity among screeners; and a mean diagnostic odds ratio of 12.3 , which was considered to be at the low end of the range for a medium level of performance (10.13-22.24). The authors concluded that this test process was well accepted by participants, demonstrated safety of screening and highlighted areas in which more training is needed. The authors of this review were in agreement that local screening programs should follow national quality assurance standards to ensure a safe and effective service [60].

\section{Cost-effectiveness of screening}

Cost-effective DR screening programs have been trialed, evaluated and fully implemented in many countries $[9,61]$. In a retrospective review of data from the US Department of Veterans Affairs, Kirkizlar et al. (2013) determined that a telemedicine program for DR screening was cost-effective only for populations $>3500$ and patients $<80$ years of age [31]. In South Africa, researchers concluded that screening patients by mobile non-mydriatic digital fundoscopy in a primary care setting was cost-effective when images were captured by a trained technician and overseen by an ophthalmic nurse and the results were read by a medical officer [62]. In a public healthcare setting in Hong Kong, investigators determined that it was cost-effective to screen patients with diabetes for DR and age-related macular degeneration in the same session [63]. In a study of cost-effectiveness of screening and laser treatment for DR and macular edema in Malawi, Vetrini et al. (2018) determined that annual photographic screening with laser treatment for STDR and macular edema was cost-effective in terms of QALYs gained, given an $80 \%$ service utilization rate [64]. The program 


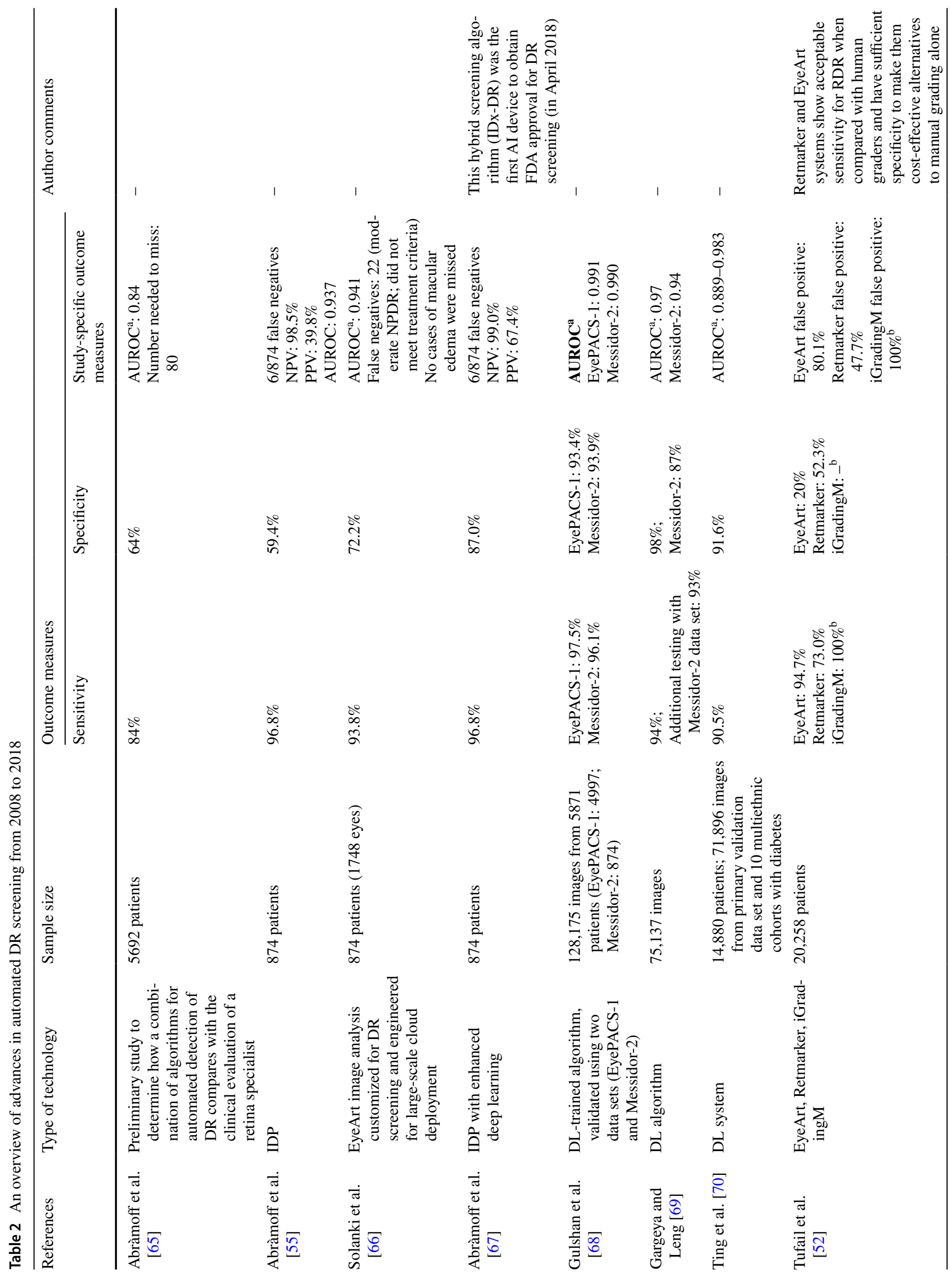




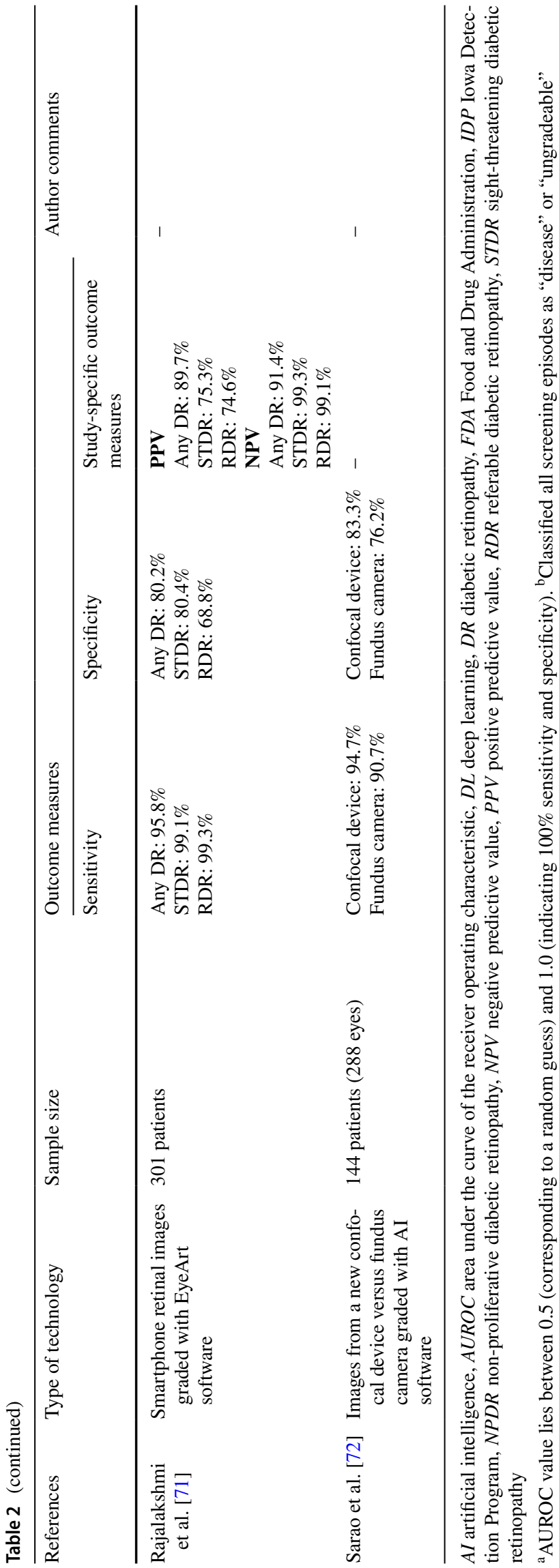

was most likely to be cost-effective when utilization was high and young patients (simulated age of 30 years) were screened [64].

Telemedicine screening systems have been cost-effective for monitoring patients with diabetes in rural and urban settings when compared with in-person screening or no screening. A retrospective chart analysis in Manitoba, Canada, which involved 4676 patients with diabetes, demonstrated average savings of Can $\$ 1007$ (approximately US\$800) per teleophthalmology examination [73]. Potential savings increased as more patients were examined [73].

\section{Screening intervals}

There is some uncertainty about the optimal interval for DR screening. Tung et al. (2008) stated that annual screening for DR was medically and economically worthwhile in a Chinese population of patients with type 2 diabetes [74]. In England, combining two consecutive years of screening data revealed that annualized rates of progression to STDR were only $0.7 \%$ and $1.9 \%$ for those with no DR at either screening or with unilateral DR at the second screening, respectively. In contrast, the annualized rate was $11 \%$ in those with bilateral DR at both screenings. The authors noted that these estimates of the risk of future development of STDR could be used to inform decisions regarding screening frequency [75].

DR screening of a hypothetical cohort in rural southern India was shown to be cost-effective from a healthcare provider perspective at 2-year intervals, but not annually [76]. From a societal perspective, telescreening was cost-effective only as frequently as every 5 years [76]. Romero-Aroca et al. [77] suggested that screening for DR every 2.5 years is costeffective, but the screening interval should be adjusted to the patient's personal risk factors. Lund et al. [78] conducted individualized risk assessments with the aim of optimizing screening intervals. They showed that DR progression could be reliably predicted and that most patients have a $<5 \%$ risk of any DR progression in a given year. The authors noted that a screening interval of 20 months could be applied to these low-risk patients [78]. Similarly, a retrospective analysis of a screening program in Turin showed that less than $4 \%$ of 4294 patients with no DR at baseline progressed to RDR over 2 years, irrespective of other clinical variables, and none required immediate treatment by an ophthalmologist [79].

Scanlon et al. [80] determined that, in the absence of personalized risk stratification, it was most likely to be cost-effective to screen all patients every 3 years; annual screening of all patients was not cost-effective. In the context of a personalized screening paradigm for STDR, screening low-risk patients every 5 years, medium-risk patients every 3 years and high-risk patients every 2 years was the most cost-effective strategy. The authors noted that although the 
algorithm should be applicable in general, more work is needed to validate screening in various populations [80].

\section{Risk factors}

DR risk has been found to be significantly associated with ethnicity, blood pressure, hemoglobin A1c (HbA1c) levels, duration of diabetes and pregnancy [35, 81]. Investigators in Portugal determined that the incidence of any DR and RDR and the DR progression rate were all related to duration of diabetes, age at diagnosis and use of insulin [82]. In a crosssectional study of four urban US sites, DR was shown to be correlated with duration of diabetes, but not with smoking status, health insurance status or knowledge of HbA1c level [83]. Risk factors associated with DR in a large screening study in Spain included duration of diabetes, HbA1c levels, blood pressure and hypertension [84]. DR is also associated with the presence of other vascular complications that occur with diabetes, and the presence of such complications should be considered when evaluating a patient's risk of the presence or progression of DR [85].

\section{Barriers to screening}

In patients with diabetes, increased time from diagnosis to first screening episode correlates with more advanced retinopathy [86, 87]. In one study, RDR was detected in $2.3 \%$ of patients screened within 6 months and in $4.2 \%$ of patients screened 3 years or more after diagnosis [86]. Therefore, screening for DR should be done promptly after diagnosis and at regular risk-based intervals thereafter.

Numerous factors are known to affect attendance at DR screening [87-91]. In an established mobile retinal screening service in Scotland, poor attendance at screening was not linked to gender or to distance from the event, but was associated with younger age, longer duration of diabetes, smoking, social deprivation, and poor HbA1c and blood pressure control; the highest proportion of non-attenders were in urban areas [92]. Findings from seven UK screening programs demonstrated that patients aged 18-34 years were least likely to promptly attend screening after registration with the screening program, and these patients had a higher risk of RDR presence at initial screening [87]. In a study of screening uptake among 21,797 patients, uptake increased with each age stratum from $67 \%$ (age 12-39 years) to $88 \%$ (age $70-79$ years) before declining to $79 \%$ in those aged $\geq 80$ years [91]. Decline in the oldest patients may have reflected age-related issues such as limited access to screening services because of comorbidity or reduced mobility. Other factors known to lower uptake include poor awareness of the importance of screening, psychological factors (such as guilt due to poor diabetic control and fear of laser treatment) and practical barriers to attendance [91]. A UK study also confirmed that uptake rates were lower in more socioeconomically deprived groups; in contrast, practices in the most socioeconomically advantaged regions had the highest uptake [93]. Moreover, there was robust disparity in uptake among the practices, which may have been due to variability in the flexibility and availability of screening appointments offered [91].

In a US study of low-income patients and their healthcare providers and staffers, investigators found a striking lack of agreement in perceived barriers to screening [90]. Providers and staffers felt that transportation, language issues, cultural beliefs or myths, denial and fear were key barriers to DR screening, whereas patients indicated that financial burden and depression were the most common barriers [90]. Judah et al. [94] evaluated the effectiveness of financially incentivizing screening among patients in the NHS Diabetic Eye Screening Programme who had not attended an appointment for at least 2 years. There was no difference in attendance between the fixed incentive and control groups (relative risk, 0.70; 95\% CI 0.35-1.39), and incentives reduced attendance compared with invitation letters in patients who were regular non-attenders. The authors emphasized that future work should focus on non-financial means of overcoming barriers to screening encountered by patients [94].

In a DR screening program at a publicly funded clinic in Alabama, only $30 \%$ of the 949 patients with diabetes adhered to interval recommendations for follow-up eye appointments [89]. The authors concluded that these programs are unlikely to meet public health goals unless adherence is promoted through adequate educational initiatives [89]. Similarly, findings of the Korean National Health and Nutrition Examination Survey indicated that only $36.3 \%$ of patients with diabetes had been screened in the previous year [95]. Patients in rural areas and those who were less educated (overall or about diabetes care) were found to be screened less often [95]. However, poor adherence has been reported in both rural and urban settings [2].

Geographic information system (GIS) mapping is useful for visualizing geographic access and barriers to eye care and may help identify underserved areas that would benefit from expansion of teleophthalmology screening programs [88]. In North Carolina, the authors used data on 1787 patients with diabetes who underwent retinal screening to develop qualitative GIS maps of patient and provider density around five telemedicine sites [88]. Results indicated that patient accessibility to healthcare professionals can be limited by geography and road networks. Primary care clinicians were somewhat uniformly distributed, but ophthalmologists were concentrated at urban centers. The authors emphasized that telemedicine has great potential for reducing barriers to care by 
connecting physicians with patients in rural, remote and underserved areas [88].

Another barrier to implementation of effective screening is delayed examination by a specialist. In a cross-sectional study in Ireland, 395 of 1542 patients were found to have some level of DR, and 11 had proliferative STDR [96]. Of these, 3 were given specialist appointments that were $>5$ months from the original referral date [96].

\section{Screening for DME}

Currently, there is a lack of evidence on screening specifically for DME; however, detection of DME is important because this condition is the leading cause of blindness in patients with type 2 diabetes [97]. One study from a DME screening program in Hong Kong demonstrated a high false positive rate $(86.6 \%)$ and a low positive predictive value (13.4\%) for screening of DME with mydriatic FP, if macular thickness was used to define the presence of macular edema [98]. This is the DME screening method currently applied in the UK and Hong Kong. The authors noted that it places large financial burdens on the healthcare system and can cause unnecessary psychological stress for patients because of the high false positive rate [98]. As noted earlier, performing OCT scans only on selected patients with suspected DME may reduce false positives and improve screening [22].

Dupas et al. [99] incorporated automated detection of DME alongside DR grading in 761 patients and noted sensitivity of $72.7 \%$ and specificity of $70.9 \%$ for DME risk. Prescott et al. [100] applied OCT alongside automated grading of fundus photographs to identify DME in 3170 patients who had positive results for DR in a prior screen involving conventional digital photography. This strategy yielded cost savings in both England and Scotland [100]. Olson et al. [101] considered the cost-effectiveness of adding OCT to a standard digital retinal photograph to detect macular edema and found that automated detection of patterns of photographic surrogate markers was superior to manual grading for detecting macular edema by OCT. The authors also determined that, by incorporating OCT into the screen prior to referral, costs could be lowered without any additional cases of macular edema being missed. They noted that worse visual acuity was associated with a fivefold higher prevalence of macular edema and therefore suggested including a functional analysis with the automated strategy [101].

\section{Summary: Fundamental principles of an effective DR screening program}

The point of care, imaging methods, use of mydriasis, training of personnel and cost-effectiveness are important factors to consider when implementing DR screening programs. Standard retinal FP is used most frequently, but other methods such as UWF may be preferable, particularly if examinations are to occur without induction of mydriasis $[17,18]$. Although no handheld device has demonstrated sensitivity and specificity comparable to seven-field examinations, such devices may be preferable from a telemedicine perspective and when barriers to standard screening exist. To reduce movement artifacts, both the handheld device and the patient's head should be fixed [24-26].

Potential barriers to screening include age (uptake rates are lowest in children and younger adults, and in older adults with reduced mobility) [91], geography [95], financial constraints or socioeconomic status [90, 91, 93] and lower education level [95]. DR screening via telemedicine is effective $[35,36]$, can improve screening by reducing patient-perceived barriers to care $[38,39,41]$, may improve follow-up [40] and appears to be cost-effective [37]. For any DR screening program to be effective, staff accreditation, appropriate IT infrastructure, continuing education and quality assurance procedures need to be in place and national standards should be followed $[4,60]$. As part of training and quality assurance, a uniform system of grading should be in place, including a definition of RDR that is understood and identified by image graders $[32,52]$. Although it is clear that using an estimate of future risk of DR, based on factors such as age and duration of diabetes, to determine screening intervals is warranted and likely to improve the cost-effectiveness of a screening program, methods for risk stratification still require standardization and validation [80].

The value of DR screening is now well established, but attempts to develop effective DME screening programs have, as yet, failed to achieve similar success [98]. However, including DME screening as part of DR evaluation may have tangible benefits [101].

Based on these considerations, the Vision Academy Working Group identified the following basic features for implementing an effective screening program for DR. We believe that these recommendations will be helpful to ophthalmology and diabetology communities.

- Examination methods must be suitable for the screening region, and DR classification/grading systems must be systematic and uniformly applied.

- Although there is variability among studies in the number of image fields acquired (ranging from one 
to nine), two-field retinal imaging is sufficient for DR screening and is preferable to seven-field imaging.

- There should be a definition of DR in place that is understood and used by screening staff to reliably identify RDR.

- In many countries/regions, screening can and should take place outside the ophthalmology clinic. Cost-effective telemedicine programs can be performed in a variety of alternative settings.

- Screening staff should be accredited and show evidence of ongoing training.

- Screening programs should adhere to relevant national quality assurance standards.

- Studies that use uniform definitions of risk to determine optimum risk-based screening intervals are required.

- Technology infrastructure should be in place to ensure that high-quality images can be stored securely to protect patient information.

- Although screening for DME in conjunction with DR evaluations may have merit, there is currently insufficient evidence to support implementation of programs solely for DME screening.

Acknowledgements The Steering Committee of the Vision Academy who advised on the publication concept included the following members: Bora Eldem, Hacettepe University, Turkey; Alex Hunyor, University of Sydney, Australia; Antonia Joussen, Charité - Berlin University of Medicine, Germany; Adrian Koh, Eye \& Retina Surgeons, Camden Medical Centre, Singapore; Jean-François Korobelnik, University Hospital of Bordeaux, France; Paolo Lanzetta, University of Udine, Italy; Anat Loewenstein, Tel Aviv Sourasky Medical Center, Israel; Monica Lövestam-Adrian, Lund University Hospital, Sweden; Rafael Navarro, Institute of Ocular Microsurgery, Spain; Annabelle A. Okada, Kyorin University School of Medicine, Japan; Ian Pearce, Royal Liverpool and Broadgreen University Hospitals NHS Trust, UK; Francisco J. Rodríguez, Fundación Oftalmológica Nacional, Colombia; Giovanni Staurenghi, University of Milan, Italy; Sebastian Wolf, University Hospital of Bern, Switzerland; and David T. Wong, St. Michael's Hospital, University of Toronto, Canada. Bayer provided financial support in the form of funding for the Vision Academy and for medical writing to Porterhouse Medical Ltd. Bayer had no role in the design or conduct of this research. The views and opinions expressed are those of the authors and the Vision Academy, and not necessarily those of Bayer.

Authors' contributions Study conception and design: Paolo Lanzetta. Acquisition of data: Paolo Lanzetta, Valentina Sarao, Peter H. Scanlon, Jane Barratt, Massimo Porta, Francesco Bandello, Anat Loewenstein. Analysis and interpretation of data: Paolo Lanzetta, Valentina Sarao, Peter H. Scanlon, Jane Barratt, Massimo Porta, Francesco Bandello, Anat Loewenstein. Drafting of manuscript: Paolo Lanzetta, Valentina Sarao, Peter H. Scanlon, Jane Barratt, Massimo Porta, Francesco Bandello, Anat Loewenstein. Critical revision: Paolo Lanzetta, Valentina Sarao, Peter H. Scanlon, Jane Barratt, Massimo Porta, Francesco Bandello, Anat Loewenstein.

\section{Compliance with ethical standards}

Conflict of interest Paolo Lanzetta has received honoraria for speaking at symposia; financial support for attending symposia; financial support for educational programs; and consultation fees from Bayer, Centervue and Novartis. Valentina Sarao has no potential conflicts of interest to declare. Peter H. Scanlon has received advisory board or speaker fees from Allergan, Bayer, Boehringer Ingelheim, Pfizer, Roche and Novartis; and support for attending meetings from Bayer. His institution has received educational research or audit grants from Allergan, Bayer, Boehringer Ingelheim, Novartis and Pfizer. Jane Barratt has no potential conflicts of interest to declare. Massimo Porta has received advisory board and speaker fees from Allergan, Novartis and SIFI. Francesco Bandello has received consultancy fees from Allergan, Bayer, Boehringer Ingelheim, Fidia, Hoffmann La Roche, Novartis, NTC Pharma, SIFI, Thrombogenics and Zeiss. Anat Loewenstein has received consultancy fees or advisory board fees from Allergan, Alcon, Bayer, Beyeonics Surgical, Neutech, Notal Vision, Novartis, Roche and Syneos Health. Her institution has received research grants/ support from Allergan, Bayer, Sensor and Novartis.

Statement of human and animal rights This article does not contain research involving human or animal subjects.

Informed consent For an expert opinion publication like this, no consent is required.

Open Access This article is licensed under a Creative Commons Attribution 4.0 International License, which permits use, sharing, adaptation, distribution and reproduction in any medium or format, as long as you give appropriate credit to the original author(s) and the source, provide a link to the Creative Commons licence, and indicate if changes were made. The images or other third party material in this article are included in the article's Creative Commons licence, unless indicated otherwise in a credit line to the material. If material is not included in the article's Creative Commons licence and your intended use is not permitted by statutory regulation or exceeds the permitted use, you will need to obtain permission directly from the copyright holder. To view a copy of this licence, visit http://creativecommons.org/licenses/by/4.0/.

\section{References}

1. Cheung N, Mitchell P, Wong TY (2010) Diabetic retinopathy. Lancet 376(9735):124-136

2. Chin EK, Ventura BV, See KY, Seibles J, Park SS (2014) Nonmydriatic fundus photography for teleophthalmology diabetic retinopathy screening in rural and urban clinics. Telemed J E Health 20(2):102-108

3. Wilson JM, Jungner YG (1968) Principles and practice of mass screening for disease. Bol Oficina Sanit Panam 65(4):281-393

4. Scanlon PH (2017) The English National Screening Programme for diabetic retinopathy 2003-2016. Acta Diabetol 54(6):515-525

5. Wong TY, Sun J, Kawasaki R et al (2018) Guidelines on diabetic eye care: The International Council of Ophthalmology Recommendations for Screening, Follow-up, Referral, and Treatment Based on Resource Settings. Ophthalmology 125(10):1608-1622

6. Gupta P, Liang Gan AT, Kidd Man RE et al (2018) Impact of incidence and progression of diabetic retinopathy on visionspecific functioning. Ophthalmology 125(9):1401-1409

7. Fenwick EK, Man REK, Gan ATL et al (2019) Beyond vision loss: the independent impact of diabetic retinopathy on 
vision-related quality of life in a Chinese Singaporean population. Br J Ophthalmol 103(9):1314-1319

8. Fenwick EK, Xie J, Pesudovs K et al (2012) Assessing disutility associated with diabetic retinopathy, diabetic macular oedema and associated visual impairment using the Vision and Quality of Life Index. Clin Exp Optom 95(3):362-370

9. Pieczynski J, Grzybowski A (2015) Review of diabetic retinopathy screening methods and programmes adopted in different parts of the world. Eur Ophthalmic Rev 9(1):49-55

10. Baeza M, Orozco-Beltrán D, Gil-Guillen VF et al (2009) Screening for sight threatening diabetic retinopathy using non-mydriatic retinal camera in a primary care setting: to dilate or not to dilate? Int J Clin Pract 63(3):433-438

11. Bragge P, Gruen RL, Chau M, Forbes A, Taylor HR (2011) Screening for presence or absence of diabetic retinopathy: a meta-analysis. Arch Ophthalmol 129(4):435-444

12. Gupta V, Bansal R, Gupta A, Bhansali A (2014) Sensitivity and specificity of nonmydriatic digital imaging in screening diabetic retinopathy in Indian eyes. Indian J Ophthalmol 62(8):851-856

13. Porta M, Boscia F, Lanzetta P, Mannucci E, Menchini U, Simonelli F (2017) Systematic screening of Retinopathy in Diabetes ( $\mathrm{REaD}$ project): an Italian implementation campaign. Eur J Ophthalmol 27(2):179-184

14. Shi L, Wu H, Dong J, Jiang K, Lu X, Shi J (2015) Telemedicine for detecting diabetic retinopathy: a systematic review and metaanalysis. Br J Ophthalmol 99(6):823-831

15. Salz DA, Witkin AJ (2015) Imaging in diabetic retinopathy. Middle East Afr J Ophthalmol 22(2):145-150

16. Goh JK, Cheung CY, Sim SS, Tan PC, Tan GS, Wong TY (2016) Retinal imaging techniques for diabetic retinopathy screening. J Diabetes Sci Technol 10(2):282-294

17. Silva PS, Horton MB, Clary D et al (2016) Identification of diabetic retinopathy and ungradable image rate with ultrawide field imaging in a national teleophthalmology program. Ophthalmology 123(6):1360-1367

18. Silva PS, Cavallerano JD, Tolson AM et al (2015) Real-time ultrawide field image evaluation of retinopathy in a diabetes telemedicine program. Diabetes Care 38(9):1643-1649

19. Hussain N, Edraki M, Tahhan R et al (2017) Telemedicine for diabetic retinopathy screening using an ultra-widefield fundus camera. Clin Ophthalmol 11:1477-1482

20. Manjunath V, Papastavrou V, Steel DHW et al (2015) Wide-field imaging and OCT vs clinical evaluation of patients referred from diabetic retinopathy screening. Eye (London) 29(3):416-423

21. Wilson PJ, Ellis JD, MacEwen CJ, Ellingford A, Talbot J, Leese GP (2010) Screening for diabetic retinopathy: a comparative trial of photography and scanning laser ophthalmoscopy. Ophthalmologica 224(4):251-257

22. Gale R, Scanlon PH, Evans M et al (2017) Action on diabetic macular oedema: achieving optimal patient management in treating visual impairment due to diabetic eye disease. Eye (London) 31(S1):S1-S20

23. Leal J, Luengo-Fernandez R, Stratton IM, Dale A, Ivanova K, Scanlon PH (2019) Cost-effectiveness of digital surveillance clinics with optical coherence tomography versus hospital eye service follow-up for patients with screen-positive maculopathy. Eye (London) 33(4):640-647

24. Bolster NM, Giardini ME, Bastawrous A (2016) The diabetic retinopathy screening workflow: potential for smartphone imaging. J Diabetes Sci Technol 10(2):318-324

25. Micheletti JM, Hendrick AM, Khan FN, Ziemer DC, Pasquel FJ (2016) Current and next generation portable screening devices for diabetic retinopathy. J Diabetes Sci Technol 10(2):295-300

26. Rajalakshmi R, Arulmalar S, Usha M et al (2015) Validation of smartphone based retinal photography for diabetic retinopathy screening. PLoS ONE 10(9):e0138285
27. Scanlon PH, Foy C, Chen FK (2008) Visual acuity measurement and ocular co-morbidity in diabetic retinopathy screening. Br J Ophthalmol 92(6):775-778

28. The Royal College of Ophthalmologists. Royal College of Ophthalmologists guidelines: screening for diabetic retinopathy. http://www.mrcophth.com/focus 1/Screening\%20for\%20Dia betic\%20Retinopathy.htm. Accessed 14 Oct 2019

29. Nasralah Z, Robinson W, Jackson GR, Barber AJ (2013) Measuring visual function in diabetic retinopathy: progress in basic and clinical research. J Clin Exp Ophthalmol 4(6):306

30. Early Treatment Diabetic Retinopathy Study Research Group (1991) Grading diabetic retinopathy from stereoscopic color fundus photographs - an extension of the modified Airlie House classification: ETDRS report number 10. Ophthalmology 98 (5 Suppl):786-806

31. Kirkizlar E, Serban N, Sisson JA, Swann JL, Barnes CS, Williams MD (2013) Evaluation of telemedicine for screening of diabetic retinopathy in the Veterans Health Administration. Ophthalmology 120(12):2604-2610

32. Wilkinson CP, Ferris FL III, Klein RE et al (2003) Proposed international clinical diabetic retinopathy and diabetic macular edema disease severity scales. Ophthalmology 110(9):1677-1682

33. The Royal College of Ophthalmologists. Diabetic Retinopathy Guidelines; December 2012. https://www.rcophth.ac.uk/wpcontent/uploads/2014/12/2013-SCI-301-FINAL-DR-GUIDE LINES-DEC-2012-updated-July-2013.pdf. Accessed 4 Mar 2020

34. Bashshur RL, Shannon GW, Smith BR, Woodward MA (2015) The empirical evidence for the telemedicine intervention in diabetes management. Telemed J E Health 21(5):321-354

35. Mansberger SL, Gleitsmann K, Gardiner S et al (2013) Comparing the effectiveness of telemedicine and traditional surveillance in providing diabetic retinopathy screening examinations: a randomized controlled trial. Telemed J E Health 19(12):942-948

36. Mansberger SL, Sheppler C, Barker G et al (2015) Long-term comparative effectiveness of telemedicine in providing diabetic retinopathy screening examinations: a randomized clinical trial. JAMA Ophthalmol 133(5):518-525

37. Nguyen HV, Tan GS, Tapp RJ et al (2016) Cost-effectiveness of a national telemedicine diabetic retinopathy screening program in Singapore. Ophthalmology 123(12):2571-2580

38. Beynat J, Charles A, Astruc K et al (2009) Screening for diabetic retinopathy in a rural French population with a mobile nonmydriatic camera. Diabetes Metab 35(1):49-56

39. Cuadros J, Bresnick G (2009) EyePACS: an adaptable telemedicine system for diabetic retinopathy screening. J Diabetes Sci Technol 3(3):509-516

40. Crossland L, Askew D, Ware R et al (2016) Diabetic retinopathy screening and monitoring of early stage disease in Australian general practice: tackling preventable blindness within a chronic care model. J Diabetes Res 2016:8405395

41. Das T, Raman R, Ramasamy K, Rani PK (2015) Telemedicine in diabetic retinopathy: current status and future directions. Middle East Afr J Ophthalmol 22(2):174-178

42. Tozer K, Woodward MA, Newman-Casey PA (2015) Telemedicine and diabetic retinopathy: review of published screening programs. J Endocrinol Diabetes. https://doi.org/10.15226 /2374-6890/2/4/00131

43. Surendran TS, Raman R (2014) Teleophthalmology in diabetic retinopathy. J Diabetes Sci Technol 8(2):262-266

44. The Queen Elizabeth Diamond Jubilee Trust Diabetic retinopathy. https://www.jubileetribute.org/our-programmes/diabe tic-retinopathy. Accessed 14 Oct 2019

45. Pareja-Ríos A, Bonaque-González S, Serrano-García M, Cabrera-López F, Abreu-Reyes P, Marrero-Saavedra MD 
(2017) Tele-ophthalmology for diabetic retinopathy screening: 8 years of experience. Arch Soc Esp Oftalmol 92(2):63-70

46. Farley TF, Mandava N, Prall FR, Carsky C (2008) Accuracy of primary care clinicians in screening for diabetic retinopathy using single-image retinal photography. Ann Fam Med 6(5):428-434

47. Romero P, Sagarra R, Ferrer J, Fernández-Ballart J, Baget M (2010) The incorporation of family physicians in the assessment of diabetic retinopathy by non-mydriatic fundus camera. Diabetes Res Clin Pract 88(2):184-188

48. Bhargava M, Cheung CY, Sabanayagam C et al (2012) Accuracy of diabetic retinopathy screening by trained non-physician graders using non-mydriatic fundus camera. Singapore Med J 53(11):715-719

49. Taylor DJ, Goatman KA, Gregory A et al (2009) Image-quality standardization for diabetic retinopathy screening. Expert Rev Ophthalmol 4(5):469-476

50. Valverde C, Garcia M, Hornero R, Lopez-Galvez MI (2016) Automated detection of diabetic retinopathy in retinal images. Indian J Ophthalmol 64(1):26-32

51. Bhaskaranand M, Ramachandra C, Bhat S et al (2016) Automated diabetic retinopathy screening and monitoring using retinal fundus image analysis. J Diabetes Sci Technol 10(2):254-261

52. Tufail A, Rudisill C, Egan C et al (2017) Automated diabetic retinopathy image assessment software: diagnostic accuracy and cost-effectiveness compared with human graders. Ophthalmology 124(3):343-351

53. Walton OB IV, Garoon RB, Weng CY et al (2016) Evaluation of automated teleretinal screening program for diabetic retinopathy. JAMA Ophthalmol 134(2):204-209

54. Soto-Pedre E, Navea A, Millan S et al (2015) Evaluation of automated image analysis software for the detection of diabetic retinopathy to reduce the ophthalmologists' workload. Acta Ophthalmol 93(1):e52-e56

55. Abràmoff MD, Folk JC, Han DP et al (2013) Automated analysis of retinal images for detection of referable diabetic retinopathy. JAMA Ophthalmol 131(3):351-357

56. De Fauw J, Keane P, Tomasev N et al (2017) Automated analysis of retinal imaging using machine learning techniques for computer vision. F1000Res 5:1573

57. Schlegl T, Waldstein SM, Vogl WD, Schmidt-Erfurth U, Langs $G$ (2015) Predicting semantic descriptions from medical images with convolutional neural networks. Inf Process Med Imaging 24:437-448

58. Fleming AD, Goatman KA, Philip S et al (2010) The role of haemorrhage and exudate detection in automated grading of diabetic retinopathy. Br J Ophthalmol 94(6):706-711

59. Fleming AD, Goatman KA, Philip S, Prescott GJ, Sharp PF, Olson JA (2010) Automated grading for diabetic retinopathy: a large-scale audit using arbitration by clinical experts. $\mathrm{Br} \mathbf{J}$ Ophthalmol 94(12):1606-1610

60. Cook S, Staff RT, Goatman KA, Olson JA, Scottish Diabetic Retinopathy Screening collaborative (2014) Quality assurance in diabetic retinal screening in South Africa. S Afr Med J 104(10):700-704

61. Pasquel FJ, Hendrick AM, Ryan M, Cason E, Ali MK, Narayan KM (2016) Cost-effectiveness of different diabetic retinopathy screening modalities. J Diabetes Sci Technol 10(2):301-307

62. Khan T, Bertram MY, Jina R, Mash B, Levitt N, Hofman K (2013) Preventing diabetes blindness: cost effectiveness of a screening programme using digital non-mydriatic fundus photography for diabetic retinopathy in a primary health care setting in South Africa. Diabetes Res Clin Pract 101(2):170-176

63. Chan CK, Gangwani RA, McGhee SM, Lian J, Wong DS (2015) Cost-effectiveness of screening for intermediate age-related macular degeneration during diabetic retinopathy screening. Ophthalmology 122(11):2278-2285

64. Vetrini D, Kiire CA, Burgess PI et al (2018) Incremental cost-effectiveness of screening and laser treatment for diabetic retinopathy and macular edema in Malawi. PLoS ONE 13(1):e0190742

65. Abràmoff MD, Niemeijer M, Suttorp-Schulten MSA, Viergever MA, Russell SR, Van Ginneken B (2008) Evaluation of a system for automatic detection of diabetic retinopathy from color fundus photographs in a large population of patients with diabetes. Diabetes Care 31(2):193-198

66. Solanki K, Ramachandra C, Bhat S et al (2015) EyeArt: automated, high-throughput, image analysis for diabetic retinopathy screening. Invest Ophthalmol Vis Sci 56:1429

67. Abràmoff MD, Lou Y, Erginay A et al (2016) Improved automated detection of diabetic retinopathy on a publicly available dataset through integration of deep learning. Invest Ophthalmol Vis Sci 57(13):5200-5206

68. Gulshan V, Peng L, Coram M et al (2016) Development and validation of a deep learning algorithm for detection of diabetic retinopathy in retinal fundus photographs. JAMA 316(22):2402-2410

69. Gargeya R, Leng T (2017) Automated identification of diabetic retinopathy using deep learning. Ophthalmology 124(7):962-969

70. Ting DSW, Cheung CY, Lim G et al (2017) Development and validation of a deep learning system for diabetic retinopathy and related eye diseases using retinal images from multiethnic populations with diabetes. JAMA 318(22):2211-2223

71. Rajalakshmi R, Subashini R, Anjana RM, Mohan V (2018) Automated diabetic retinopathy detection in smartphone-based fundus photography using artificial intelligence. Eye (London) 32(6):1138-1144

72. Sarao V, Veretti D, Lanzetta P. Screening performance of an automated image analysis software for the detection of diabetic retinopathy using a conventional fundus photography or a confocal white LED device: a comparison study. Abstract presented at the Association for Research in Vision and Ophthalmology (ARVO) 2019 Annual Meeting; Vancouver, Canada, 28 April-2 May 2019

73. Kanjee R, Dookeran RI, Mathen MK, Stockl FA, Leicht R (2017) Six-year prevalence and incidence of diabetic retinopathy and cost-effectiveness of tele-ophthalmology in Manitoba. Can J Ophthalmol 52(Suppl 1):S15-S18

74. Tung TH, Shih HC, Chen SJ, Chou P, Liu CM, Liu JH (2008) Economic evaluation of screening for diabetic retinopathy among Chinese type 2 diabetics: a community-based study in Kinmen, Taiwan. J Epidemiol 18(5):225-233

75. Stratton IM, Aldington SJ, Taylor DJ, Adler AI, Scanlon PH (2013) A simple risk stratification for time to development of sight-threatening diabetic retinopathy. Diabetes Care 36(3):580-585

76. Rachapelle S, Legood R, Alavi Y et al (2013) The cost-utility of telemedicine to screen for diabetic retinopathy in India. Ophthalmology 120(3):566-573

77. Romero-Aroca P, de la Riva-Fernandez S, Valls-Mateu A et al (2016) Cost of diabetic retinopathy and macular oedema in a population, an eight year follow up. BMC Ophthalmol 16:136

78. Lund SH, Aspelund T, Kirby $P$ et al (2016) Individualised risk assessment for diabetic retinopathy and optimisation of screening intervals: a scientific approach to reducing healthcare costs. Br J Ophthalmol 100(5):683-687

79. Porta M, Maurino M, Severini S et al (2013) Clinical characteristics influence screening intervals for diabetic retinopathy. Diabetologia 56(10):2147-2152

80. Scanlon PH, Aldington SJ, Leal J et al (2015) Development of a cost-effectiveness model for optimisation of the screening 
interval in diabetic retinopathy screening. Health Technol Assess 19(74):1-116

81. Errera MH, Kohly RP, da Cruz L (2013) Pregnancy-associated retinal diseases and their management. Surv Ophthalmol 58(2):127-142

82. Dutra Medeiros M, Mesquita E, Gardete-Correia L et al (2015) First incidence and progression study for diabetic retinopathy in Portugal, the RETINODIAB study: evaluation of the screening program for Lisbon region. Ophthalmology 122(12):2473-2481

83. Owsley C, McGwin G Jr, Lee DJ et al (2015) Diabetes eye screening in urban settings serving minority populations: detection of diabetic retinopathy and other ocular findings using telemedicine. JAMA Ophthalmol 133(2):174-181

84. Rodriguez-Poncelas A, Miravet-Jiménez S, Casellas A et al (2015) Prevalence of diabetic retinopathy in individuals with type 2 diabetes who had recorded diabetic retinopathy from retinal photographs in Catalonia (Spain). Br J Ophthalmol 99(12):1628-1633

85. Pearce I, Simó R, Lövestam-Adrian M, Wong DT, Evans M (2019) Association between diabetic eye disease and other complications of diabetes: implications for care. A systematic review. Diabetes Obes Metab 21(3):467-478

86. Scanlon PH, Aldington SJ, Stratton IM (2014) Delay in diabetic retinopathy screening increases the rate of detection of referable diabetic retinopathy. Diabet Med 31(4):439-442

87. Scanlon PH, Stratton IM, Leese GP et al (2016) Screening attendance, age group and diabetic retinopathy level at first screen. Diabet Med 33(7):904-911

88. Jani PD, Forbes L, McDaniel P, Viera A, Garg S (2017) Geographic information systems mapping of diabetic retinopathy in an ocular telemedicine network. JAMA Ophthalmol 135(7):715-721

89. Keenum Z, McGwin G Jr, Witherspoon CD, Haller JA, Clark ME, Owsley C (2016) Patients' adherence to recommended follow-up eye care after diabetic retinopathy screening in a publicly funded county clinic and factors associated with follow-up eye care use. JAMA Ophthalmol 134(11):1221-1228

90. Lu Y, Serpas L, Genter P, Anderson B, Campa D, Ipp E (2016) Divergent perceptions of barriers to diabetic retinopathy screening among patients and care providers, Los Angeles, California, 2014-2015. Prev Chronic Dis 13:E140

91. Moreton RBR, Stratton IM, Chave SJ, Lipinski H, Scanlon PH (2017) Factors determining uptake of diabetic retinopathy screening in Oxfordshire. Diabet Med 34(7):993-999

92. Leese GP, Boyle P, Feng Z, Emslie-Smith A, Ellis JD (2008) Screening uptake in a well-established diabetic retinopathy screening program: the role of geographical access and deprivation. Diabetes Care 31(11):2131-2135

93. Scanlon PH, Carter SC, Foy C, Husband RF, Abbas J, Bachmann MO (2008) Diabetic retinopathy and socioeconomic deprivation in Gloucestershire. J Med Screen 15(3):118-121

94. Judah G, Darzi A, Vlaev I et al (2017) Incentives in Diabetic Eye Assessment by Screening (IDEAS) trial: a three-armed randomised controlled trial of financial incentives. Health Serv Delivery Res 5(15)

95. Byun SH, Ma SH, Jun JK, Jung KW, Park B (2013) Screening for diabetic retinopathy and nephropathy in patients with diabetes: a nationwide survey in Korea. PLoS ONE 8(5):e62991

96. McHugh S, Buckley C, Murphy K et al (2013) Qualityassured screening for diabetic retinopathy delivered in primary care in Ireland: an observational study. Br J Gen Pract 63(607):e134-e140

97. Romero-Aroca P, Sagarra-Alamo R, Basora-Gallisa J, BasoraGallisa T, Baget-Bernaldiz M, Bautista-Perez A (2010) Prospective comparison of two methods of screening for diabetic retinopathy by nonmydriatic fundus camera. Clin Ophthalmol 4:1481-1488

98. Wong RL, Tsang CW, Wong DS et al (2017) Are we making good use of our public resources? The false-positive rate of screening by fundus photography for diabetic macular oedema. Hong Kong Med J 23(4):356-364

99. Dupas B, Walter T, Erginay A et al (2010) Evaluation of automated fundus photograph analysis algorithms for detecting microaneurysms, haemorrhages and exudates, and of a computerassisted diagnostic system for grading diabetic retinopathy. Diabetes Metab 36(3):213-220

100. Prescott G, Sharp P, Goatman K et al (2014) Improving the costeffectiveness of photographic screening for diabetic macular oedema: a prospective, multi-centre, UK study. Br J Ophthalmol 98(8):1042-1049

101. Olson J, Sharp P, Goatman K et al (2013) Improving the economic value of photographic screening for optical coherence tomography-detectable macular oedema: a prospective, multicentre. UK study. Health Technol Assess 17(51):1-142

Publisher's Note Springer Nature remains neutral with regard to jurisdictional claims in published maps and institutional affiliations. 